\title{
PERVIVENCIAS CANANEAS (UGARÍTICAS) EN EL CULTO FENICIO - III
}

GREGORIO DEL OLMO LETE

Universidad de Barcelona

$B^{\complement} L H M N$

En otro lugar ' hemos argüido de pasada en favor de la relación entre fenicio $h m n$ y ugarítico $h m n$, con el resultado de que el Nombre Divino $\mathrm{B}^{\iota} \mathrm{l} \mathrm{Hmn}$ significaría «Baal (del) Hamón» $\left(^{*} B a^{c} l u\right.$ Hamāni) o "Baal del santuario palatino", de acuerdo con el valor que el lexema tiene en la literatura cultual de Ugarit. La argumentación filológica y la información bibliográfica allí apuntadas, sobre todo en relación con los testimonios epigráficos e iconográficos del arameo $h \mathrm{hn}^{2} /$ *hamānă, han sido recogidas de nuevo, desarrolladas y completadas por Drijvers ${ }^{2}$ en favor del valor apuntado de «santuario, edícula cultual, capilla». Su amplia proliferación en toda la

1 Cf. G. DEL Olmo LETE, "La 'capilla' o 'templete' (hmn) del culto ugarítico», Aula Orientalis 2 (1984) 278, n. 9 (con bibliografia). Entre los autores que sostienen la equiparación de fen. hmmn con ug. hmn cabe destacar a F. M. Cross, Canaanite Myth and Hebrew Epic (=CMHE), Cambridge, Mass. 1973, p. 27, que otorga al lexema el valor toponímico de Monte Amanus. Desconoce, con todo, la presencia de ug. hmn en los textos cultuales y recoge sólo sus ocurrencias como elemento onomástico (cf. infra nn. 6 y 9); en el mismo sentido, LIPIŃsKI (cf. infra n. 14); y también, aunque más impreciso, G. Garbini, oA 7 (1968) 157 ss. (vid. P. Magnanini, Le iscrizioni fenicie dell'Oriente [= IFO], Roma 1973, pp. 18-20).

${ }^{2}$ Cf. H. J. W. DRIJVERS, "Aramaic hmn' and Hebrew hmn", JSS 33 (1988) 165-179. La etimología propuesta, *hmh, 'proteger', era ya conocida (así Elliger; cf. DEL OLMO LETE, art. cit., p. 277, n. 2). De ser cierta tal etimología (cf. árabe/ar. "hamã), el lexema arameo sería ditinto de ug. hmn (cf. *hmm en ar. y ug.). Pero éste constituye un 'dato' histórico-religioso, la etimología es mera 'especulación'. La equiparación de fen. hmn con ug. hmn lleva más bien hacia una etimología derivada de "hw/ym; cf. Cross, op. cit., p. 27, n. 81; DEL OlMo LeTE, art. cit., p. 278; DE MOOR - SPRONK, UF 14 (1982) 160-161, n. 65, pero todavía en UF 20 (1988) 168 traduce DE MOOR b'l kmn por "Baal-of-the-Hearth", al margen, por tanto, de tal equiparación. Esta no se impone, de todos modos, necesariamente desde el punto de vista linguístico, como muy bien advierte F. O. Hvidberg-Hansen, La Déesse TNT, Copenhague 1979, p. 128; pero el valor histórico-religioso la apoya decididamente. 
región siria, cuyos monumentos la testimonian a partir del siglo I a. C. como elemento de culto típicamente semítico ${ }^{3}$, remite por fuerza a una tradición cultual previa de ese estrato de población. Se presenta así como la 'democratización' del culto palatino cananeo del bmn en Ugarit, atestiguado hasta fines del siglo II d. C., en este otro extremo del ámbito evolutivo, del que fenicio h̆mn, hebreo hammān, representa la fase central durante la que se fue fraguando el proceso evolutivo semántico y funcional: "capilla o santuario palatino-dinástico / dinástico-poliade / poliade-particular».

Tal argumentación, que no vamos a repetir aquí, exluye que $b c l$ hmn pueda relacionarse con la base *hmm, "arder, quemar", de donde $\mathrm{hmm}>$ "crematorio, altar de incienso", opinión ampliamente aceptada en medios filológicos e imperturbablemente repetida por los historiadores del mundo fenicio-púnico. Pero ni los datos epigráficos (sobre todo las inscripciones palmirenas) ni los iconográficos (representaciones del $\mathbf{h m} \boldsymbol{n}^{2}$ en monumentos palmirenos, nabateos y púnicos) autorizan otorgar a $\mathrm{hmn}$ tal valor semántico, como la documentación aducida por mí y la desarrollada por Drijvers dejan bastante claro. A través de la misma se puede seguir, a la vez, la paulatina evolución de las opiniones de los semitistas al respecto. Nadie discute la presencia y función de los 'altares de incienso' en el culto fenicio-púnico, como la arqueología testimonia, sino su relación específica con Baal hasta el punto de constituir el determinativo de su título o nombre específico, tratándose como se trata de un instrumento cultual de universal y antigua raigambre oriental.

Por otra parte, la presencia de tal Nombre Divino en la inscripción de Kilamuwa ${ }^{4}$ y posteriormente en otra de Umm el-‘Amed/ 'Amāwid ' han sugerido con insistencia un valor toponímico para hmm, valor que también se había supuesto ya al relacionarlo con

${ }^{3}$ Cf. Drijvers, art. cit., pp. 175 ss. Téngase en cuenta que en Palmira perdura también el culto de Bel-Ham(m)ón; cf. J. TeIXIDOR, The Pantheon of Palmyre, Philadelphia 1979, pp. 12-13; ID. The Pagan God. Popular Religion in the GrecoRoman Near East, Princeton N. J. 1977, p. 127 ss., n. 75 (también, según Teixidor: 'Lord of the Amanus'); HvidBerg-HANSEN, op. cit., p. 18, n. 85.

4 Cf. H. Donner - W.ROLlig, Kanaandische und Aramaische Inschriften (= KAI), Wiesbaden 1962-1964, n 24:16; IFO, pp. 45-47; Y. AvisHUR, Ketovot phinikiyot wehaMigra', I/II (= KPM), Yerusalayim 1979, pp. 205-214; J. C. L. GiBson, Textbook of Syrian Semitic Inscriptions. Volume 3: Phoenician Inscriptions (= TSSI 3), Oxford 1982, $\mathrm{n}^{2} 13: 16$.

5 Cf. M. Dunand - R. Duru, Umm el 'Amed. Texte, Paris 1962, p. 192; IFO, pp. 16-17; TSSI 3, $\mathrm{n}^{2}$ 32:1-2. 
hitita-hurrita Ham(m)ani/Hammunni, el monte o sierra del Amanus ${ }^{6}$. Otro valor toponímico impreciso (localidad en las proximidades de Tiro) se ha otorgado también últimamente al determinativo en la mentada inscripción de Umm el-cAmed y en otra de la próxima localidad de Ma'șūb ?.

Pero la primera de estas ecuaciones ha de ser descartada por razones lingüísticas, aunque histórico-religiosamente la 'divinización' de tal montaña es perfectamente admisible en aquellas culturas ${ }^{8}$. Si fonológicamente en el sistema silábico está justificada la alofonía $\nu / h$, en el sistema consonántico semítico-occidental tal topónimo se transcribe amn (*'mn), como certifica el testimonio ugarítico y avalan las mismas transcripciones silábicas hititas y hurritas ${ }^{9}$. Se trata, por lo demás, de un topónimo sobradamente relevante como para suponer que la notación semítica es anterior a estas transcripciones de uno u otro tipo ${ }^{10}$.

${ }^{6}$ Cf. Del Olmo LeTe, art. cit., p. 279, n. 9. Así opinan, por ejemplo, Cross, op. cit., p. 28, n. 86 (vid. supra n. 1); LIPIŃsKI (vid. infra n. 14); TEIXIDOR (vid. supra n. 3); BordreuIl (vid. infra n. 13); también HvidBerg-HANSEN, op. cit., pp. 127-128, que sin negar el valor $\mathrm{hmn}=$ Amanus, lo excluye para fen. (b'l) $\mathrm{hmm}$.

${ }^{7}$ Cf. KAI, no 19:3-4; IFO, pp. 17-18 TSSI 3, ne 31:3-4; CAQUOT, Semitica 15 (1965) 30; BORDREUIL, Studia Phoenicia IV (vid. infra n. 12), p. 84.

${ }^{8}$ Tal posibilidad no es tenida en cuenta por el editor de los textos hurritas de Ugarit que mencionan un (in) hmn (KTU 1.116:6,1 16; 1.135:7; 1.60:6; 1.128:10; 181.42:51 (?); CTA 177:8-9, 12); cf. E. LAROCHE, en Ugaritica V, Paris 1968, pp. 519 ss., 535 (sin traducción); ID., Glossaire de la langue hourrite, Paris 1980, 91 ('sens inconnu'; cf. también p. 113); HVIDBERG-HANSEN, op. cit., p. 102, n. 325. Para una bibliografia sobre el tema cf. DEL Olmo LeTE, art. cit., pp. 278-279. nn. 9 y 16; Cross, op. cit., p. 28.

${ }^{9}$ Cf. KTU 2.33:16: g̀r amn (cf. GORDON, UT, $n^{2}$ 226); hb. 'amand, pún.-gr. amoun, 'amanós (cf. CROSS, CMHE, pp. 27 ss., para las referencias); hit. HUR.SAG a-m(m)ana, hur. HUR.SAG a-ma-na-hi (cf. F. DEL MONTE - J. TISCHLER, Die Orts- und Gewdissernamen der hethitischen Texte (Répertoire Géographique des Textes Cunéiformes, Band 6), Wiesbaden 1978, pp. 13 ss. ('Das Amanos-Gebirge'). La pretensión de Cross (art. cit., p. 27) de ver en esta transcripción la designación de otra montaña «in the same general region» no tiene fundamento. Comete además un paralogismo al citar en su apoyo (n. 81) a Lewy, quien relaciona las lecturas silábicas hamanu con la base *hmm y su pretendida divinidad solar *Hammu (a este propósito of. H. B. Huffmon, Amorite Personal Names in the Mari Texts, Baltimore MA 1965, p. 197: "Lewy is certainly wrong"). Por otra parte, ac.-ug. PPNN ab-di-ha-ma-ni, IR-ha-ma-nu (RS 16.200:3; 16.348:6') corresponden a ug. 'bdlomn (cf. KTU 4.75:I 3; 4.170:14; 4.177:3; 4.332:12; para la variante abdhmn de RIH 83/5:20 cf. P. BORDREUIL, SEL 5 (1988) 26); de ser cierto aquí el valor toponímico de bmn, se esperaria la transcripción "bdamn, de acuerdo con lo dicho.

${ }^{10}$ La transcripción acádica del topónimo es ambigua, como en general la de todo lexema de primera 'alef (' 1 ), en los diversos dialectos y épocas. Las transcripciones ha-ma-mu/i y similares son neoasirias (cf. W. VON SODEN, GAG, pp. 24 ss.; $R$. BORGER, Die Inschriften Asarhaddons Königs von Assyrien (BAfO 9), Berlin 1956, 
Por otro lado, aparte de esa equiparación lingüisticamente equivocada, la primera de esas interpretaciones se ha guiado por otra no menos equivocada equiparación que ha pretendido hacer de $b^{c} l$ hmmn el correlato del $b^{c} l s p n$. Pero spn no es un topónimo geográfico empírico sino un topónimo de geografia mítica " que cada ciudad o reino 'localiza' desde su propia perspectiva: los habitantes de Ugarit, en el Monte Casio (buršan bazzi, Gebel el-Aqrac), los primitivos cananeos, al parecer, en el Monte Hermón, los judíos, en Sión, los habitantes del Bajo Egipto, en la frontera asiática del Delta ${ }^{12}$. Es decir, Sapānu/Saphón no es sin más indefectiblemente el Monte Casio/buršan Hazzi, ni desde luego está probado que así lo fuera para los 'fenicios' del siglo I, una vez desaparecida la ciudad de Ugarit ${ }^{13}$.

En línea con la propuesta aquí defendida creemos que, efectiva-

p. 131) y neobabilónicas (cf. R. ZADOK, Geographical Names According to New- and Late-Babylonian Texts (RGTC, 8), Wiesbaden 1985, p. 148 (una " $\mathrm{r}$ " $a-m / b a-n u$ se la sitúa probablemente «not far from Uruk»). Para la antigua transcripción acádica cf. D. O. Edzard - G. Farber - E. Sollberger, Die Orts und Gewässernamen der präsargonischen und sargonischen Zeit (RGTC, 1), Wiesbaden 1977, p. 11 (Amanum: ama-nam sa-dū GIŠ.EREN; ama-a-núm hur-sağ-eren-ta, "das Zedergebirge Amanus»); a la inversa, para variantes en la transcripción silábica $(A-, H-)$ de $/ \mathbb{C} / \mathrm{y} / \mathrm{h} / \mathrm{cf}$. HUFFMON, op. cit., p. 166. Un lexema con $/ \mathrm{h} /$ originaria difícilmente tendría una transcripción distinta en silábico; las variantes de transcripción se explican si su primera consonante es un $/ \%$. Pero no se puede excluir en absoluto una retrotranscripción en hur. consonántico con $/ \mathfrak{h} /$ a partir del uso escribal acádico (asirio), no obstante el propio uso silábico (cf. supra n. 9). En todo caso, es claro que ug. hmn de los textos cultuales (KTU 1.106:12-14; 1.112:3, 8; RIH 77/2B:1) nada tiene que ver con el Monte Amanus.

11 Sobre el tema puede verse últimamente N. WyATT, "Sea and Desert: Symbolic Geography in West Semitic Religious Thought», UF 19 (1987) 375-389; e interesantes sugerencias también en J. TEIXIDOR, "Géographies du voyageur au Proche-Orient ancien", AuOr 7 (1989) 105-115.

12 Sobre las diversas localizaciones y tradiciones de Saphón, dejando aparte la interpretación de b'l hmn como 'Baal del Amanus', véase E. LIPIŃSKI, «El's Abode. Mythological Traditions Related to Mount Hermon and the Mountains of Armenia", OLP 2 (1971) 13-69. P. BORDREUIL, "Attestations inédites de Melqart, Baal Hiamon et Baal Saphon à Tyr", Studia Phoenicia IV (Religio Phoenicia), Namur 1986, p. 83; C. Bonnet, "Typhon et Baal Saphon», Studia Phoenicia V (Phoenicia and the East Mediterranean in the First Millenium B.C.), Leuven 1987, pp. 101-143; MARGALIT, OLP 19 (1988) 90.

13 BordreulL, (art. cit., p. 85) percibe bien el problema aquí mencionado en relación con Tiro, pero ofrece una solución (identificación con el Saphón meridional) que no estimo acertada y que depende de su previa identificación de Baal Hamón como 'Baal del Amanus'. La objeción ya habla sido planteada por Gsell y Leglay; cf. HVIDBERG-HANSEN, op. cit., p. 128. 
mente, $b c l h m n$ es el correlato de $b^{c} l s p n$, pero en cuanto ambas denominaciones señalan la 'morada urbana' y 'mitológica' de Baal, de su santuario celeste y terrestre, como rey de los dioses y rey de la ciudad, titular del nuevo culto dinástico-poliade. Se trata así de dos advocaciones paralelas. Desde este punto de vista ${ }^{14}$ cabe interpretar las diversas inscripciones en que aparece el sintagma $b^{c} \mathbf{l} \mathbf{h m n}$ antes de convertirse en la advocación clásica de la divinidad titular y tutelar de los tophet-s púnicos, excluyendo su pretendido valor como nombre de montaña o de ciudad.

\section{a) Inscripción de Kilamuwa is}

Esta inscripción regia, redactada en el más puro 'estilo de palacio', acaba con una fórmula imprecatoria contra quien la dañe:

wmy ysht hspr z

$y$ šht $r^{2} s^{y}$

b'l smd 's lgbr wysht r's

$b^{\complement l}$ hmn 'š lbmh $w r k b^{\prime} l b^{c} l b t$ "y si alguno estropea esta inscripción, que le rompa la cabeza

Baal-Samd, el de GBR, y que le rompa la cabeza

Baal-Hamón, el de BMH, y Rakkabel, el jefe de la dinastía».

14 Cf. a este propósito E. LIPIŃsKI, "Zeus Ammon et Baal-Hammon», Studia Phoenicia IV, p. 329: “Il est cependant apparu, à la lumière des textes d'Ugarit, que hamman n'est pas un encensoir ou quelque autre ustensile rituel, mais un lieu de culte situé sur une hauteur, peut-être le succédané liturgique de la montagne sainte»; (vid. también Hvidberg-Hansen, op. cit., p. 128). Pero en la página precedente interpreta el sintagma como relativo al monte Amanus en la misma dirección que Cross, disociando las bases *hmn y "hmn (cf. nn. 1 y 11); en cambio, la cita de Xella nada dice de tal correlación ug.-fen.; cf. también E. LIPIŃSKI, BiOr 41 (1984) 439.

15 Cf. KAI, n 24:15-16; IFO, pp. 46-47; KPM, pp. 205-214; TSSI 3, n 13 (con bibliografia); últimamente J. C. DE MOOR, "Narrative Poetry in Canaan", UF 20 (1988) 165-171; S. D. SPERLING, "KAI 24 Re-examined", UF 20 (1989) 323-337, que no estudia 1. 16. Las inscripciones de Kilamuwa reflejan en general la clásica ideología regia de Canaán; cf. J. L. R. Wood, Kingship at Ras Shamra-Ugarit. A Study of the Literary, Ritual, and Administrative Documentation, Diss. 1972, McMaster University; y últimamente, L. M. MuNTINGH, "The Conception of Ancient SyroPalestinian Kingship in the Light of Contemporary Royal Archives with Special Reference to Recent Discoveries at Tell Mardikh (Ebla) in Syria", en T. MikAsA (ed.), Monarchies and Socio-religious Traditions in the Ancient Near East, Wiesbaden 1984, pp. 1-10; N. WyatT, "The Hollow Crown: Ambivalent Elements in West Semitic Royal Ideology», UF 18 (1986) 421-436. 
La imprecación, paralela a la conocida fórmula ugarítica ${ }^{16}$, invoca la intervención de las divinidades ancestrales dinásticas y hace de $b^{\circ} l$ hlmn el dios tutelar de BMH, anterior rey de Y'DY. Ahora bien, en este contexto dinástico es del todo improbable que el dios tutelar sea la divinidad titular de otra ciudad, en este caso, el de una oscura ciudad al sur de Tiro, sin especial significación en la historia política de la Fenicia. Si se atiende a las determinaciones de los otros dioses invocados, se aprecia su clara raigambre cananea, en las que el apelativo $b c l$ mantiene aún su valor nominal "señor de...». En tal sentido $b c l$ smd, "el señor de la maza», como muy bien recoge Gibson, recuerda el arma mágica que Kothar proporciona a Baal y que le garantiza su victoria sobre el dios Yam. Análogamente, b`l hmn es "el señor del *Hamon» o templo palatino que la literatura cultual de Ugarit menciona como santuario del culto regio-dinástico, el $i l b t$; del mismo modo que Rakkabel es $b c l b t$, «el señor o jefe de la dinastía" " que se recomienda por sí misma y que enlaza directamente con la mitología y el culto regio atestiguado en Ugarit.

\section{b) Inscripción de Umm el-cAmed XIII ${ }^{18}$}

Inscripción votiva sobre el pedestal de la estatua de una esfinge. En este caso no podemos precisar si ésta iba enmarcada dentro del consabido templete de columnas que con tanta frecuencia rodea las

${ }^{16}$ Cf. KTU 1.16 VI 54-57 (1.2 I 7-8): « ¿Que rompa Horanu, oh hijo, que rompa Hóranu tu cabeza, 'Attartu, nombre de Baclu, tu cráneo!» (cf. infra ne 37). Para una fórmula semejante véase últimamente S. LACKENBACHER, "Une nouvelle attestation d'Ištar Hurri dans un contrat trouvé à Baniyas (Syrie)», en J. M. DURAND - J. R. KuPPER, (eds.), Miscellanea Babylonica (Fs. Birot), Paris 1985, pp. 153 ss.: «A quien altere estas palabras que Ashtarte de las tumbas y el dios de la casa (DINGIR sa E) le aniquilen".

17 Ambas expresiones aparecen en los textos cultuales de Ugarit: il bt, bclt bhtm (1.39:13; 1.102:1; 1.115:3, 7, 9; cf. XELLA, TRU I, pp. 386 ss.; y n. precedente). La equivalencia entre $i l b t$ y $b \bullet l$ bt se aprecia igualmente en las inscripciones siguientes, que alternan $i l / b$ ' l hmn (CROss, CMHE, p. 24, n. 60); la denominación 'l hmn aparece en otras inscripciones de Umm el-'Amed como atributo de Milki'ašstart. En todo caso, un rey que se llamaba Bmh es normal que tuviera por dios dinástico a $B^{<} l \mathrm{Hmn}$ (cultual), lo mismo que Gbr tenía por tal a $B^{c} l$ Smd (guerrero). Se trata de dos dinastias distintas de la de Kilamuwa, cuyo dios dinástico era $R k b$ l; cf. GIBSON, TSSI 3, p. 39; W. F. Albright, Yahweh and the Gods of Canaan, London 1968, p. 203.

18 Cf. M. Dunand - R. DuRu, Umm el 'Amed. Texte, Paris 1962, pp. 181 ss. (?); A. CAQUOT, "Le dieu Milk'ashtart et les inscriptions de 'Umm el 'Amed», Semitica 15 (1965) 29 s8.; IFO, p. 22; TSSI 3, n² 32. 
representaciones iconográficas en las estelas. El texto ofrece evidentes ecos de la ideología regia cananea:

l'dny lmlk'strt 'l hmn

kprt hrs mtm

'š $y$ tn 'bdk

'bd'dny bn 'bd'lnm

bn 'štrt'zr b'l ḥmn

$k m^{2} s^{y} l^{2} \operatorname{lnm}$

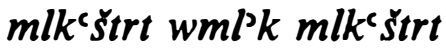

$k s m^{\circ} q l$

ybrk
«A mi señor, a Milki`aštart, dios del Hamón, propiciatorio (?) de oro macizo (?)

que ha hecho tu siervo 'Abdadoni, hijo de 'Abdilonim, hijo de 'Aštarti'azar, dueño/patrón del Hamón ${ }^{19}$, como su ofrenda a los dioses Milki`aštart y Ángel de Milki`aštart porque escucharon su voz. ¡Que de(n) su bendición!».

Entendida de esta manera, Milki`aštart es seguramente la divinidad poliade del lugar y en consecuencia la titular de su santuario regio-urbano («dios del Hamón»), encarnación del rey-dios, del Milku cananeo que tenía su morada en 't trt (hebreo 'Aštarôt ${ }^{20}$. El oferente se presenta como el "patrón del Hamón" con lo que éste parece asumir el carácter de santuario o capilla particular. Ahora bien, el carácter privado de algunos santuarios ( $\left(\mathrm{hm}^{2} / \mathrm{mqdS}\right.$ ) queda patente en inscripciones nabateo-palmirenas e incluso púnicas ${ }^{21}$; el uso parece estar también atestiguado en Ugarit ${ }^{22}$. Pero podría muy bien ser que el donante fuera el dinasta del lugar y así el patrón oficial de su santuario. Por otro lado, la presencia del «Ángel de Milki'aštart» enlaza directamente con la divinidad 'mensajera' que

19 En el caso de que el texto dijera bbcl, la traducción correspondiente sería «en cuanto duen̂o/patrón del Hamón", referido preferentemente al oferente más que a la divinidad, de la que ya se dijo que era il hmn (1. 1) y a la que se vuelve a hacer referencia a continuación; cf. TSSI 3, p. 122. Por su parte IFO, p. 22: “miembro del HMN".

${ }^{20}$ No se puede hablar de 'divinidad compuesta' (cf. TSSI 3, p. 39; CAQUOF, art. cit., p. 32); la documentación ugarítica ha cambiado la consideración de este problema y la identificación de esta divinidad. Cf. S. RibichinI - P. Xella, «MilkcAstart, MLK(M) e la tradizione siropalestinese sui Refaim" RSF 7 (1979) 145-158; G. DEL Olmo Lete, «Los nombres divinos de los reyes de Ugarit», AuOr 5 (1927) 60 ss.

${ }^{21}$ Sobre todo en las inscripciones que nos hablan de 'donación', o incluso 'construcción', de 'santuarios'; cf. por ejemplo, TSSI 3, n 42 (Pyrgi); KAI n 72 (Ibiza), 140 (Gebel Mansur); DrIJVERS, JSS 33 (1988) 166 ss. (inscripciones palmirenas); Swiogers en Studia Phoenicia I-II (Sauvons Tyr / Histoire phénicienne), Leuven 1983, pp. 125 ss. (a propósito de $K A I, \mathrm{n}^{\circ} 17 ;$ TSSI 3, $\mathrm{n}^{2} 30$ ).

${ }^{22}$ Cf. M. Yon, en G. Roux (ed.), Temples et sanctuaires, Lyon 1984, pp. 47 ss. 
acompaña en Ugarit a las grandes divinidades, y más en concreto nos remite a un texto como KTU 1.124 , de sentido regio-adivinatorio, donde aparece(n) 'mansajero(s)/ángel(es)' (mlakk) en relación con la consulta cultual acerca de la curación (?) de un 'infante'. Esa actividad 'oracular' los textos cultuales ugaríticos la ponen en relación con la actuación regia en el bmn ${ }^{23}$. De nuevo esta inscripción fenicia nos sitúa así en el mundo de las concepciones religiosas cananeas.

\section{c) Inscripción de Umm el-cAmed IV (Ma'șub) ${ }^{24}$}

Inscripción fechada en 222 a. C., lo que nos aproxima enormemente a la época de la atestiguada difusión del hamānā palmireno. La construcción de un pórtico en torno al mismo, que aquí se menciona, nos remite curiosamente a similares estructuras de tales edificios cultuales como han sido determinadas por Drijvers ${ }^{25}$. Reproducimos a continuación las líneas que dicen al caso:

'rpt kbrt ms'sms wsply
's bn h'lm mlk'strt
$w^{c} b d y$ b'l hmn
$k^{\prime} s t r t$
$b^{\prime}$ 'srt 'l hmn
$\ldots \ldots . .$.
$k m^{\prime} s$ bn
'yt 'hry h[mqds'm
's b'rs
«Pórtico occidental con sus ... que edificó el dios Milkicaštart y sus siervos los patrones del Hamón a 'Aštart en el recinto del dios del Hamón

como edificaron los demás santuarios que hay en el país,

La figura de un dios que coadyuva a la construcción de un santuario no tiene nada de excepcional en la concepción oriental, como pone bien de manifiesto Caquot ${ }^{26}$. Pero de nuevo entendemos que los b'l hmn no son los "ciudadanos de Hamón", sino los «dueños o patrones» de tal estructura cultual dentro del complejo

${ }^{23}$ Cf. KTU 1.106:14; 1.112:8. En general, las parejas de dioses dióscoros-mensajeros ( $q d s$-amrr, gpn-ugr), sobre todo țkmn-snm, revelan la importancia de los 'ángeles' en la mitologia y religión cananea; cf. últimamente D. PARDEE, «Tukamuna wa Sunama", UF 20 (1988) 195-199. De nuevo la interpretación de IFO, p. 22, es divergente: «i chierici di Milkçstart».

24 Cf. KAI n² 19; IFO, pp. 17-18; TSSI 3, $\mathrm{n}^{2} 31$; CAQUOT, Semitica 15 (1965) 31 ss.; del Olmo LeTe, AuOr 2 (1984) 279, $\mathrm{n}^{2}$ 9; Bordreull, Studia Phoenicia IV, p. 84.

${ }^{25}$ Cf. Drijvers, art. cit., pp. 175 ss.

${ }^{26}$ Cf. CAquor, art. cit., p. 32. 
sacro del "dios del Hamón», que según se deduce de la inscripción precedente, no es otro que Milki ‘aštart ${ }^{27}$. Lo que nos lleva a suponer que $m l^{b} k$ mlkcstrt es un título regio: se refiere al rey como lugarteniente de la divinidad poliade y constructor, con sus súbditos, de "todos los demás santuarios del país". 'Aštart, por su parte, es posiblemente la diosa paredra, vista así ya en el nombre compuesto del dios titular, una vez olvidada quizá la original significación toponímica del segundo elemento, a pesar de que la tardía tradición deuteronomística hebrea aún la conocía.

\section{d) Amuleto de Tiro ${ }^{28}$}

Documento epigráfico de reciente aparición (1982) en la región de Tiro y datable en el siglo VI, es decir, de la misma zona pero mucho más antiguo que las inscripciones citadas anteriormente de Umm el-cAmed. El editor, que acepta el sentido de "ciudadanos de Hamón" para b`l hmn en aquéllas, prefiere para ésta el de "Baal del Hamón/Amanus». A priori resulta dudosa tal anfibología.

$\begin{array}{ll}l b^{c} l \text { hmn } & \text { "A Baal-(del)-Hamón } \\ \text { wlbclspn } & \text { y a Baal-(del)-Saphón. } \\ \text { kybrknn } & \text { ¿Que ellos me bendigan!» }\end{array}$

Tenemos aquí yuxtapuestas las dos advocaciones, que nosotros entendemos desde la polaridad mencionada más arriba de «morada urbana/mítica" de la divinidad, no desde una polaridad toponímica, bien se sitúe spn en el Gebel el-Aqra', bien en el delta del Nilo, como prefiere Bordreuil, que señala la incongruencia de la primera identificación (la más 'segura', en cambio, desde el punto de vista histórico-religioso), si b`l hmn significa "Baal del Amanus»: "on saisit mal la raison d'une vénération conjointe de deux divinités voisines l'une de l'autre». La dificultad desaparece si $\mathrm{hmn}$ no puede indicar el Amanus.

${ }^{27}$ La interpretación of recida de $w^{c} b d y$ b $/$ hmn tiene posiblemente su paralelo en el ya citado NP ugarítico 'bd bmn (cf. supra n. 9). En todo caso, es insostenible la interpretación del ND mlkcstrt que ofrece GiBson (cf. supra n. 20). El nombre del dios aparece en otras dos inscripciones de Umm el-AAmed; cf. IFO, pp. 16-17.

${ }^{28}$ Cf. BORDREUIL, art. cit., pp. 82 ss. 
e) Dedicatoria-tipo de Cartago ${ }^{29}$

Es conocida la diversidad de destinatarios que ofrecen las dedicatorias púnicas: $l b^{c} l / b^{c} l \mathrm{hmn} / b^{c} l ' d r, l b c l$ wltnt, ltnt wlbcl hmn. Esta última variante es la más frecuente en las dedicatorias de Cartago y es la que aquí seleccionamos, pero es claro que el valor del determinativo es extensible a las demás variantes.

lrbt lint pn bel

wl'dn lbcl hmn

'š ndr $X$ bn $X$

hksme $q l^{\text {l }}$

$y b r k^{2} / b r k^{2}$
"A la señora, a Tinnit, rostro de Baal, y al señor, a Baal-(del)-Hamón, voto que hizo $X$ hijo de $X$, porque escucharon su voz. ¿Que le den su bendición!».

Como divinidad titular del santuario poliade y tutelar de la dinastía, Baal es el dios protector por excelencia del mundo cananeo. Pero sobre todo, como dios de la vida, que muere y resucita, "que da la vida al que le invita y agasaja» (cf. KTU 1.17 VI 30ss), con cuyo vigor y fuerza son investidos los "reyes eternos" (KTU 1.108:23ss), es el destinatario ineludible de todo rito en el que de alguna manera esté implicado el problema de la vida y su restauración, como pensamos es el caso del sacrificio $m l k$, según lo expuesto en otra parte ${ }^{30}$.

Creemos que esta interpretación, además de garantizar la continuidad histórico-religiosa del término $\mathrm{hmn} / \mathrm{hmn}$, soslaya una equiparación sin garantía filológica ( $\mathrm{hmn}=$ Amanus) y una interpretación lingüísticamente forzada, como la de $b c l \mathrm{hmn}$, «ciudadanos de Hamón", desconocido lugar fenicio que, de hallarse presente en el nombre divino mentado, asumiría la titularidad religiosa del gran dios del mundo púnico. Este valor toponímico haría difícilmente explicable la aparición de tal advocación en ese ámbito durante el primer milenio a. C. Un dios de 'otro lugar' dificilmente podría llegar a ser el dios de Cartago y sus colonias. Sólo un valor religioso genérico lo haría transferible como advocación divina de

29 Cf. entre los varios estudios sobre esta fraseología estereotipada la síntesis de M. G. Amadasi Guzzo, "La documentazione epigrafica dal Tofet di Mozia e il problema del sacrificio Molk», Studia Phoenicia IV, pp. 193 ss.; ID., Scavi a Mozia Le iscrizioni, Roma 1986, pp. 45 ss.; también F. BERTRANDY - M. SzNYCER, Les stèles puniques de Constantine, Paris 1987, pp. 81 ss.

30 Cf. G. DEL Olmo Lete, «Pervivencias cananeas (ugariticas) en el culto fenicio II ('El culto mlk')» [en prensa, Fs. Sznycer]. 
cualquier ciudad, de acuerdo con su funcionalidad cultual. Éste sería nuestro caso, si se acepta la interpretación propuesta. Es lo mismo que aconteció con una advocación como mlqrt, con la que conviene $b c l$ hmn en muchos aspectos. Es en realidad, como ésta, una denominación genérica: «Rey de la ciudad», "Patrón del santuario palatino", que como tal se aplica en primera instancia al rey empírico y pasa luego a ser una denominación del rey 'divino' como titular del santuario dinástico.

La divinidad titular del santuario palatino es, como divinidad tutelar de la ciudad, una divinidad poliade y dinástica del tipo $m l k$ y como tal puede ser advocación divina de diferentes ciudades (como $b^{c} l s p n$ ), con valor propio en cada una, dentro del modelo de religión poliade típico de las ciudades fenicio-púnicas del primer milenio a. C. ${ }^{31}$. Baal-(del)-Hamón de Cartago es así la figura compleja que aúna el aspecto regio-divino de $m l k$ con el revitalizador de $b$ cl, como Melqart, Ešmún y Adonis ${ }^{32}$. Unificación que ya se da en la mitología cananea: Baal es el mlk por antonomasia en el que se incorporan y de quien reciben su vigor eterno todos los mlkm. Su paredra será Tinnit, como 'Aštart lo fue de su 'predecesor' en Cartago, Melqart ${ }^{33}$. Una vez asumida esta advocación como la del dios tutelar poliade de Cartago (!), su difusión por el mundo púnico sería un hecho asegurado; aunque probablemente tal advocación, como propia de la divinidad del sacrificio $m l k$, fue traída directamente de Fenicia y aparece en establecimientos pre-púnicos del

${ }^{31}$ Cf. S. Ribichini, Poenus Advena. Gli fenici e l'interpretazione classica, Roma 1985, pp. 43 ss. ('L'eroe divino').

32 En realidad todas estas divinidades (o mejor, advocaciones del mismo tipo de divinidad) aparecen en un momento u otro como 'dios de Cartago', ciudad en la que, dada la peculiar fluctuación del sistema político, la concepción del 'rey divino' poliade resulta más imprecisa y cambiante.

${ }^{33}$ ¿Tienen que ver la implantación de su culto y la consiguiente suplantación (?) de Melqart con los cambios políticos habidos en Cartago en el s. IV, que es cuando comienza a aparecer la pareja Tht - Bel Hmn en las dedicatorias de sus estelas?; cf. Amadasi Guzzo, art. cit., p. 196. Sobre el posible sentido político del culto a Tinnit véase M. E. AuBET, Tiro y las colonias fenicias de Occidente, Barcelona 1987, p. 137: "Melqart, como dios dinástico, simbolizó la monarquía, en tanto que Tanit y Baal simbolizaron la oligarquía, representada por la familia de los Magónidasw; también, citando las opiniones de Charles-Picard y Garbini al respecto, ID., El santuario de Es Cuieram, Ibiza 1982, pp. 41 ss., donde parece implicarse lo contrario en relación con la significación política de los Magónidas. En todo caso, no puede desconocerse la especificidad del culto a que van unidas tales divinidades ( $m l k)$. 
Mediterráneo occidental ${ }^{34}$. Es posible, asimismo, que con el correr del tiempo tal advocación perdiera su sentido original y se atribuyesen a $\mathbf{~ h m n}$ otros valores, confundiéndolo tal vez con otros homógrafos-homófonos.

$\check{S} M / P N B^{K} L$

La designación de la diosa 'Aštart como šm bcl y la de Tannit/Tinnit como $p n$ bcl son un prominente ejemplo de pervivencia religiosa en el ámbito cananeo-fenicio. Ambas desarrollan aspectos bien conocidos de la ideología del 'nombre' y la 'presencia' divinas operantes en aquél ${ }^{35}$.

La primera, cštrt šm $b c l$, que aparece en la inscripción de Ešmunazar en el s. IV ${ }^{36}$, es conocida por una fórmula imprecatoria que nos transmite la mitología ugarítica en la segunda tablilla del ciclo de Baal-Anat ${ }^{37}$, un milenio antes como mínimo. Pero, además, esta persistencia de la designación, explicable en principio por su uso mágico-cultual, tiene su refrendo precisamente en la misma tablilla mentada, único lugar de esa mitología donde se recurre al 'nombre' de Baal como motivo de su proceder. Es la misma diosa 'Aštart la que «por el nombre le reprocha: 'vergüenza debías tener...'» (bšm tg'rm 't trt bt...), dado su comportamiento desmesurado con su enemigo cautivo vencido ${ }^{38}$. Ella, que poco antes (KTU 1.2 I 40) había tratado de contenerle y le había asimismo reprochado su innoble comportamiento con los mensajeros, es la hipóstasis de tal 'nombre', que actúa como la defensora de su prestigio, del 'buen nombre de Baal'. Conocido es el eco que estos theologoúmena del 'buen nombre' y la 'vergüenza' poseen en la lírica sacra hebrea ${ }^{39}$.

${ }^{34}$ Así lo presupondria, por ejemplo, la estela de Malta; cf. TSSI 3, ne 21-22, p. 73.

33 Cf. Cf. Cross, CMHE, p. 30, infra n. 39.

${ }^{36}$ Cf. $K A I n^{2} 14: 18$; IFO, pp. 3 ss.; TSSI 3 n $^{\circ} 28: 18$; también, al parecer, en Palmira; cf. Hvidberg-HaNSEN, La Déesse TNT, p. 18, n. 85.

$37 \mathrm{Cf}$. KTU 1.2 I 8, texto fragmentario pero perfectamente reconstruible, como fórmula hecha, desde KTU 1.16 VI 56.

${ }^{38}$ Cf. KTU 1.2 IV 28; cf. J. C. DE MOOR, The Seasonal Pattern in the Ugaritic Myth of BaGlu, Neukirchen-Vluyn 1971, pp. 127, 139 ss.; G. DEL. Olmo LeTE, Mitos y Leyendas de Canaán, Madrid 1981, pp. 113, n. 121, y 177; para una traducción diferente, que no ha encontrado aceptación, of. HVidberg-HaNSEN, op. cit., p. 106, n. 6.

39 Para una exposición sintética cf. E. JENNI - C. WestermanN, Diccionario teológico manual del Antiguo Testamento, I/II. Madrid 1978/1985. I, cols. 399-402; II, cols. 1.173-1.207; también II, cols. 548-582 para el tema del 'rostro/presencia' divina. Para otras interpretaciones-etimologías de $\$ m b s l$ cf. HvidBerG-HANSEN, op. cit., p. 107, n. 15. 
Esta misma ideología se precisa en otros textos ugaríticos, como aquel que nos habla de la 'proclamación del nombre' del dios Yammu, de su nuevo nombre 'regio', por parte del dios supremo Ilu, como condición previa de su ascensión al trono de los dioses ${ }^{40}$. Por su parte, Kôtaru, cuando quiere proporcionar a $B a^{c} l u$ un arma mágica definitiva, 'proclama' también su nombre destructor, que significa su efecto y que por eso la convierte en tal ${ }^{41}$. Aparte de estas exaltaciones del 'nombre', curiosamente concentradas en las dos primeras tablillas del ciclo de Baal-Anat, el 'nombre' del dios Ilu es objeto de celebración celeste por parte de los 'héroes divinos', los rapauma, que le asisten ${ }^{42}$.

El uso de tal theologoúmenon está confirmado igualmente por la onomástica, que nos ofrece nombres propios como šmbnš, šmbll, šmlbu/i, šmlšn, šmmlk, šmmn, šmnt (acadio šu-um-a-na-tu), šmrg, šmrm ${ }^{43}$.

Por su parte, el atributo $p n b c l$, dicho de Tinnit, es un correlato del anterior y expresa, como aquél, la irradiación de la presencia divina sobre el fiel, de la que su paredra se convierte en hipóstasis. Del mismo modo como la divinidad 'hace valer su nombre' en favor de sus fieles, según la conocida expresión bíblica, del mismo modo hace 'brillar su rostro' sobre el que se le acerca en demanda de ayuda. La expresión posee incluso un uso 'civil', dicha del soberano que acoge la demanda de su súbdito ${ }^{*}$. Como en el caso anterior, también en éste la onomástica nos proporciona un uso similar con nombres como pnmn (y acadio pa-ni-ya-nu, pa-ni-la, pa$n i-i s-t a-a){ }^{45}$.

Pero es de nuevo la mitología la que nos desarrolla el theologoúmenon. En la oscura teogonía que nos presenta el texto KTU 1.12 I

${ }^{40}$ Cf. KTU 1.1 IV 15; Del Olmo LeTe op. cit., pp. 100 ss.

${ }^{41} \mathrm{Cf}$. KTU 1.2 IV 11, 18.

${ }^{42} \mathrm{Cf} . \mathrm{KTU} 1.22$ I 6.

${ }^{43}$ Cf. F. GrÓndahl, Die Personennamen der Texte aus Ugarit, Roma 1967, pp. 193 ss., 355, 414.

${ }^{44}$ Cf. KTU 2.16:9: wpn sps' $n r$ ly, "y la faz del 'Sol' ha brillado en mi favor"; carta de Talmiyanu a la reina de Ugarit, su madre, informándole de sus gestiones ante el rey de los Hititas, el 'Sol'.

${ }^{45}$ Cf. GrOndahl, op. cit., pp. 47, 173, 327, 347, 405; los textos KTU 1.9:13 (pn hdd) y 1.25:6 (pn ym) son desgraciadamente fragmentarios y no permiten una interpretación adecuada. El determinativo divino pn $b \triangleleft l$ pervive en monedas de Ascalón e inscripciones de Palmira; cf. Hvidberg-HaNSEN, op. cit., pp. 17-18, nn. 76-85. 
9ss, la naturaleza divina de los recién nacidos queda fijada, en primer lugar también en este caso, por la 'proclamación de su nombre' que hace su padre, el dios $I l u$, y revelada además por el aspecto baálico que ofrecen:

bhm qrnm $\mathrm{km}$ trm wgbtt $\mathrm{km}$ ibrm whhm pn bel "tendrán cuernos como toros, y 'morrillo' como morlacos, y tendrán también el rostro de $B a^{\varsigma} l u$ ".

(KTU 1.12 I 30-33)

Sólo así, equipados con un 'rostro' igual, pueden resultar contrincantes válidos del dios de la fertilidad y la vida, al que el dios supremo, síntesis interpretativa de la ambigüedad de la existencia con sus fuerzas antagónicas y su perenne contraste vida-muerte de las que él es 'padre', no cesa de poner a prueba, en este caso en un tono incluso socarrón. Recuérdese su apoyo no disimulado a Yammu, Môtu y 'Ațtaru como contrincantes de Baclu. También estos nuevos dioses del 'desierto' tienen 'rostro' como el de $B a^{\varsigma} l u$ y por eso pueden (momentáneamente) superarle. Pero al fin, el 'rostro' que resplandece y triunfa en la mitología cananea es el 'rostro' de $B a^{\varsigma} l u$, del que la diosa Tinnit será la hipóstasis.

Durante mucho tiempo se pensó que era ésta una divinidad específicamente púnica, por su masiva aparición en las inscripciones cartaginenses a partir del siglo IV ${ }^{46}$. Pero la sucesiva comparecencia de tal nombre divino en documentos epigráficos de la Fenicia metropolitana, en Sarepta-Sarafand y otros lugares ${ }^{47}$, la revelan como una diosa sin duda fenicia del primer milenio.

Incierta es, en cambio, su equiparación con otras advocaciones del panteón cananeo en el que ella no aparece. Mientras su diferenciación de 'Aštart resulta asegurada por su comparecencia conjunta como doble advocación diferenciada ${ }^{48}$, no aparece clara su equipa-

${ }^{46}$ Cf. supra n. 33.

47 Cf. P. BordreuIL, «Tanit du Liban (Nouveaux documents phéniciens III)», Studia Phoenicia V, Leuven 1987, pp. 79-85.

${ }^{48}$ Cf. KAI n. 81:1: Irbt Kstrt wltnt blbnn. 
ración con Ațiratu ${ }^{49}$ ni con 'Anatu ${ }^{30}$. Se trata, en todo caso, de advocaciones y epifanías de un mismo tipo de divinidad femenina, paredra de $B a^{c} l u$, que encarna los rasgos esenciales de diosa de la fertilidad y la guerra que arrastra ya desde la tradición babilónica en torno a la diosa Inanna/Ištar. Sin embargo, lingüísticamente, tanto 'Anatu como Tinnit se presentan como innovaciones propias del área semítica occidental, lo que autoriza a buscar en ese ámbito su génesis lingüística, la 'etimología' de su nombre. El ejercicio en sí no tiene mayor transcendencia, a no ser que permita descubrir en la misma la persistencia, de un theologoumenon operante en la mitología cananea. Y así como para el nombre divino ' $n t$ se han propuesto diversas etimologías a partir de bases semíticas claras (*cyn/ $n y){ }^{51}$, que 'explican' el sentido del mismo dentro del marco de relaciones y concepciones que aquella divinidad supone, cabría intentar lo mismo a propósito de tnt en relación con alguna otra base ${ }^{52}$.

Estas advocaciones se presentan lingüísticamente como atributos nominales ('strt šm bcl, tnt pn b`l, 'nt ' $g l t b c l, n k l$ ar yrb), por lo que la interpretación predicativa del tipo atrt ym, 'la que pisa sobre el

49 Es posible que se diera una cierta 'equiparación' secundaria en tal sentido, teniendo en cuenta la previa 'conflación' de las figuras de El y Baal, y consiguientemente de sus 'consortes'. Tal 'conflación' se aprecia en la Biblia Hebrea, en la que confluyen aquellas figuras en la de Yahweh, así como en los panteones fenicios del primer milenio, en los que la figura de El tiene escasa relevancia frente a las de los dioses 'baálicos', y en la interpretatio púnico-romana del propio b'l hmn; cf. HVIDBERGHansen, La Déesse TNT, Copenhague 1979, pp. 43, 117 y passim. Pero la argumentación en favor de la equiparación Tinnit/Ashera aportada por CROss (CMHE, pp. 31 ss.) no me parece decisiva; cf. al respecto HvidBERG-HANSEN, op. clt., pp. 115-119; LIPIŃSKI, BO 45 (1988) 388 ss., en contra de la equiparación propuesta por W. A. MAIER, ASerah: Extrabiblical Evidence, Atlanta GA 1986.

${ }^{50}$ Como quiere F. O. Hvidberg-Hansen, op. cit. pp. 139 ss., que ve en el nombre de la diosa fenicia (antes de conocerse su ocurrencia en textos provenientes de la metrópoli) la transposición del 'nt a través de la epéntesis de un prefijo líbico-bereber $t$ - que induciría la caída/asimilación de la primera radical semítica (!). Se trata de un modelo de monografia exhaustiva con una conclusión equivocada.

51 Cf. entre otros, A. S. KAPELRUD, The Violent Goddess. Anat in the Ras Shamra Texts, Oslo 1969, pp. 27 ss.; J. C. DE MoOR, UF 1 (1969) 224; P. J. VAN ZIJL, Baal. A Study of Texts in Connection with Baal in the Ugaritic Epics, Neukirchen-Vluyn 1972, p. 65; R. DU MESNIL DU BuIsSON, Berytus 26 (1978) 65, n. 66; J. GRAY, UF 11 (1979) 321-322, n. 42; HVIDBERg-HANSEN, op. cit., pp. 14 ss., 103 ss., 139 ss.

52 Se ha relacionado con el sintagma $n / y t n$ pnm, «don, mirada, gloria de Baal»; para esta y otras etimologias cf. Hvidberg-Hansen, op. ctt., pp. 27-28, 138. Por su parte Cross, op. cit., p. 32 ss. lee el nombre como tannittu, userpentinan, relacionado con tannin (cf. HVIDBERG-HANSEN, op. cit., p. 116; LIPIŃsKI, art. cit., p. 389. 
mar' ${ }^{53}$, tiene escasa verosimilitud. En cambio, el carácter nominal de tnt se refleja quizá en la variante $t m t$, que atestiguan algunas inscripciones ${ }^{54}$, claro indicio de que la advocación ya no se entendía en su sentido 'etimológico', lo que favorecía esta alofonía/alografia.

Es posible que en un momento posterior este nombre divino se relacionase 'etimológicamente' con la frecuente designación de la ofrenda como mtnt en las múltiples fórmulas votivas de las estelas fenicio-púnicas en las que aparece tht al lado de su pareja b`l hmn ${ }^{55}$. Como tal, Tinnit viene a ocupar en la religión púnica el lugar que la mitología cananea otorgaba a Anat, al margen de toda posible relación lingüística entre sus nombres.

\section{RESUMEN}

Continuando estudios previos dedicados a poner de manifiesto el continuum cultural cananeo-fenicio, sobre todo en el ámbito del culto, se analizan en éste el ND $b^{c} l \mathbf{h m n}$ y los apelativos $\mathrm{sm} / \mathrm{pn}$ b $l$. Para estos dos últimos casos la pervivencia mitológica es universalmente admitida y aquí se aportan datos literarios que la resaltan. En cuanto a $b^{\iota} l \mathrm{hmn}$, se propone su vinculación con ug. hmn, lo que lleva a entender el ND como designación de divinidad palatina y dinástica, excluyendo su referencia a determinativos toponímicos, como generalmente se interpreta.

\section{SUMMARY}

Going on with previous researches devoted to show the Canaanite-Phoenician cultural continuum, especially in the field of worship, the ND $b^{c} l \mathrm{hmn}$ and the appellatives $5 \mathrm{~m} / p n b^{c} l$ are analyzed here. Mythological survival is universally accepted in these two last cases, and literary information which emphasizes it is provided in this work. Concerning $b^{c} l h m n$, its connection with ug. $h m n$ is suggested, and this leads to an understanding of ND as designation of a dynastical and palatine deity, excluding its reference to toponymical determinatives, as it is generally interpreted.

53 Sobre esta opinión de Albright y otras cf. Hvidberg-Hansen, op. cit., pp. 7980; para una nueva sugerencia cf. MARGALIT, OLP 19 (1988) 86, n. 26; ID., VT 39 (1989) 374.

${ }^{54}$ Cf. BordreuIL, art. cit., pp. 82 ss.; para posibles valores de pún. tmt cf. C. F. JeAn - J. Hoftuzzer, Dictionnaire des inscriptions sémitiques de l'ouest, Leiden 1965, p. 330. Pero no me parece obligado ver en esa grafia el nombre de la diosa Tinnit.

s5 Cf. los trabajos citados supra en n. 29. 